\title{
DISCOVERY OF QPO FROM X PERSEI WITH RXTE
}

\author{
TOSHIAKI TAKESHIMA \\ NASA Goddard Space Flight Center, \\ Universities Space Research Aassociation \\ takeshim@ginpo.gsfc.nasa.gov
}

$\mathrm{X}$ Persei is a Be X-ray binary pulsar with $~ 835$-sec pulsation period[1]. Different from most other Be X-ray binaries, X Persei does not exhibit Xray outbursts. A binary period of $\sim 580$ days has been suggested[2], but is not confirmed the by follow-up observations.

The Proportional Counter Array (PCA) onboard Rossi X-ray Timing Explorer (RXTE) observed X Persei twice on March 19 and April 1,1996 . Since a couple of flares in the timescale of $\sim 100$-sec were observed whose pulse phases are not constant, we removed such flares from data for the analysis of the coherent pulsation. Applying the folding technique to these data, the barycentric corrected pulse period are obtained as $840 \pm 1.8$ and 837.6pm 2.5 seconds for March and April data, respectively. The most plausible averaged pulse period for all data is $838.91 \mathrm{pm} 0.04 \mathrm{sec}-$ onds for the epoch of $\mathrm{MJD}=50617.9311$, while the possibility for the period of $838.15 p m 0.04$ or $839.41 p m 0.04$ seconds cannot be rejected completely.

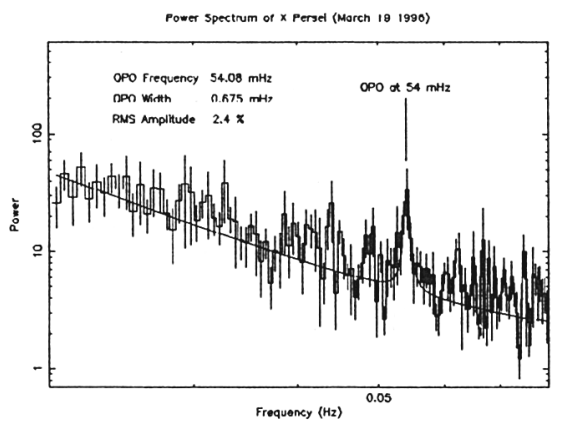

Figure: Power spectrum of $\mathrm{X}$ Persei around QPO frequency range (20-80 $\mathrm{mHz}$ ). Histogram with error bars is the data and the solid line is the best fit model.

By calculating the power spectra for two observations separately, we found a broad peak structure at around $54 \mathrm{mHz}$ from March 19 data. The figure shows the power spectrum between $20-80 \mathrm{mHz}$ with the best fit model. The employed model was the sum of a power law component (for continuum), a Lorentzian (for QPO), and a constant (for Poisson noise). The addition of a QPO component (three parameters) reduces the $\chi^{2}$ value by 14.4 (from 175.5 to 161.1 ) for the d.o.f. of 120 . From the F-test, the addition of a QPO component 
is significant at the $99.5 \%$ level. The centroid frequency, the width (FWHM), and the root-mean-square (RMS) amplitude of QPO are $54.08_{-0.28}^{+0.23} \mathrm{mHz}$, $0.67_{-0.25}^{+0.23} \mathrm{mHz}$, and $2.4 \pm 1.0 \%$ (90\% confidence), respectively.

QPO from X-ray binary pulsars have been reported from seven sources to date. These pulsars have relatively short to moderate pulse periods of 0.7-103 seconds and are believed to be disk-fed pulsars. The QPO frequencies concentrate to $10-200 \mathrm{mHz}$. Since the QPO observed from X Persei have typical frequency, width and amplitude of QPO from X-ray binary pulsars, it is natural to think that QPO from X Persei have the same origin as those observed from other X-ray binary pulsars.

The origins of QPO not only from X-ray binary pulsars but from lowmass X-ray binaries and black hole binaries are still unclear. The beat frequency model (BFM)[3] is generally regarded as the most plausible model for QPO from X-ray binary pulsars. In the BFM, QPO originate from the inhomogeneity of the accreting matter at the inner edge of the accretion disk. In other words, if the BFM works, the detection of QPO is evidence of the existence of an accretion disk. On the other hand, the facts that $\mathrm{X}$ Persei is a low luminosity Be $\mathrm{X}$-ray binary system, the Roche lobe is not filled, and that the random-walk like behavior of pulse period change in relatively short time scale similar to Vela $X-1$ is known, strongly suggest that X Persei is a wind-fed system. If so, this is the first detection of QPO from a wind-fed pulsar.

Assuming the BFM and the accretion disk model for binary pulsars by Ghosh and Lamb[4,5], and using the observed QPO frequency $(54.1 \mathrm{mHz})$, pulsation frequency $(1.2 \mathrm{mHz})$, and X-ray luminosity $\left(1.1 \times 10^{34} \mathrm{erg} / \mathrm{sec}\right)$, the strength of the surface magnetic field is estimated as $1.7 \times 10^{11}$ Gauss. This value is relatively small for a binary X-ray pulsar. E-cut energy is thought to be roughly proportional to the strength of the magnetic field[6]. From this relation, the estimated strength of the surface magnetic field is $0.64-1.15 \times 10^{12}$ Gauss, which is by 3.8-6.8 times larger than that derived by assuming the BFM and Ghosh \& Lamb's model. The moderate pulse modulation of $\mathrm{X}$ Persei $(\sim 50 \%)$ does not support the very weak magnetic field, either.

\section{References}

[1] White et al. 1976, Monthly Notices Roy. Astron. Soc., 176, 201.

[2] Hutchings et al. 1974, Astrophys. J. Letts., 191, L101.

[3] Alper \& Shaham 1985, Nature, 316, 239.

[4] Ghosh and Lamb 1979a, Astrophys. J., 232, 259.

[5] Ghosh and Lamb 1979b, Astrophys. J., 234, 296.

[6] Makishima \& Mihara 1992, Frontiers of X-ray Astronomy, Proc. of the Yamada Conf. p23. 